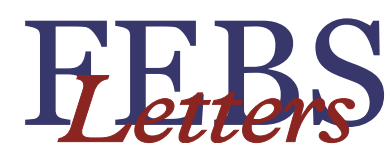

journal homepage: www.FEBSLetters.org

Review

\title{
TERRA biogenesis, turnover and implications for function
}

\author{
Sascha Feuerhahn ${ }^{1}$, Nahid Iglesias ${ }^{1}$, Andrea Panza ${ }^{1}$, Antonio Porro ${ }^{1}$, Joachim Lingner ${ }^{*}$ \\ Swiss Institute for Experimental Cancer Research (ISREC), School of Life Sciences, Frontiers in Genetics National Center of Competence in Research, \\ Ecole Polytechnique Fédérale de Lausanne (EPFL), Station 19, 1015 Lausanne, Switzerland
}

\section{A R T I C L E I N F O}

\section{Article history:}

Received 1 July 2010

Revised 19 July 2010

Accepted 20 July 2010

Available online 23 July 2010

Edited by Wilhelm Just

\section{Keywords:}

TERRA

Non-coding RNA

Telomere

Telomerase

\begin{abstract}
A B S T R A C T
Telomeres are heterochromatic structures at the ends of eukaryotic chromosomes. As other heterochromatin regions, telomeres are transcribed, from the subtelomeric region towards chromosome ends into the long non-coding RNA TERRA. Telomere transcription is a widespread phenomenon as it has been observed in species belonging to several kingdoms of the eukaryotic domain. TERRA is part of telomeric heterochromatin in addition to being present in the nucleoplasm. Here, we review the current knowledge of TERRA structure, biogenesis and turnover. In addition, we discuss presumed roles of this RNA during replication of telomeric DNA, heterochromatin formation and the regulation of telomerase.
\end{abstract}

() 2010 Federation of European Biochemical Societies. Published by Elsevier B.V. All rights reserved.

\section{Introduction}

Telomeres, the physical ends of eukaryotic chromosomes, are associated with specialized proteins that protect the natural DNA ends from being recognized as sites of DNA damage [1]. During semiconservative DNA replication, telomeres shorten due to the end replication problem and to chromosome end processing by nucleases. Telomere shortening is counteracted by telomerase, a ribonucleoprotein enzyme that can extend the $3^{\prime}$ ends of chromosomes by reverse transcribing a small region of its tightly associated RNA moiety [2-4]. The progressive erosion of telomeres and the activation of telomerase are linked to chromosomal stability, cellular immortalization, and tumor progression $[5,6]$. Reactivation of telomerase in human cancer cells allows overcoming the senescence barrier and is a key requisite of cancer cells to attain unlimited proliferation potential.

Despite the fact that telomeres are heterochromatic structures, recent studies have demonstrated that eukaryotic telomeres are transcribed into TElomeric Repeat containing RNA (TERRA) [710]. The nuclear localization of TERRA and its enrichment at telomeres indicates that TERRA is not translated but that it functions as non-coding RNA in the nucleoplasm and in telomeric and subtelomeric heterochromatin structures. Several roles of TERRA have been postulated [11]. TERRA may act as a regulator of the telomeric heterochromatin in analogy to other long non-coding RNAs like

\footnotetext{
* Corresponding author. Fax: +41216930720.

E-mail address: joachim.lingner@epfl.ch (J. Lingner).

1 These authors contributed equally and are listed alphabetically.
}

Xist and HOTAIR [11]. Telomeric DNA provides a challenge for the replicative machinery and TERRA may under certain circumstances become an obstacle and interfere with the semiconservative replication machinery. TERRA transcription may regulate telomerase globally or at individual telomeres as this RNA can act as a direct inhibitor of the telomerase enzyme.

\section{TERRA primary and higher order structure}

It has been demonstrated for human cells and the yeast Saccharomyces cerevisiae that TERRA is transcribed at several if not all chromosome ends from the subtelomere towards the end of the chromosomes [7,9]. All human TERRA and most yeast TERRA 5' ends contain a 7-methylguanosine $\left(\mathrm{m}^{7} \mathrm{G}\right)$ cap structure (Porro, A., Feuerhahn, S., Reichenbach, P. and Lingner, J., submitted for publication; Panza, A. and Lingner, J., unpublished) (Fig. 1A). This indicates that TERRA $5^{\prime}$ ends are defined by transcription initiation and are not due to post-transcriptional processing events. TERRA is heterogeneous in length, ranging from 100 to $>9000$ bases in mammals [7]. In S. cerevisiae, TERRA is also heterogeneous with an average length of 380 bases [9]. The telomeric tract-derived 5'-UUAGGG-3' repeats of the vast majority of human TERRA molecules have an approximate length of 200 bases (Porro, A., Feuerhahn, S., Reichenbach, P. and Lingner, J., submitted for publication). Thus, the majority of TERRA sequence stems from the subtelomeric region and size heterogeneity is largely due to different lengths of this part of the RNA. Most human TERRA molecules terminate with the sequence UUAGG-3' (Porro, A., Feuerhahn, S., Reichenbach, P. and Lingner, J., submitted for 

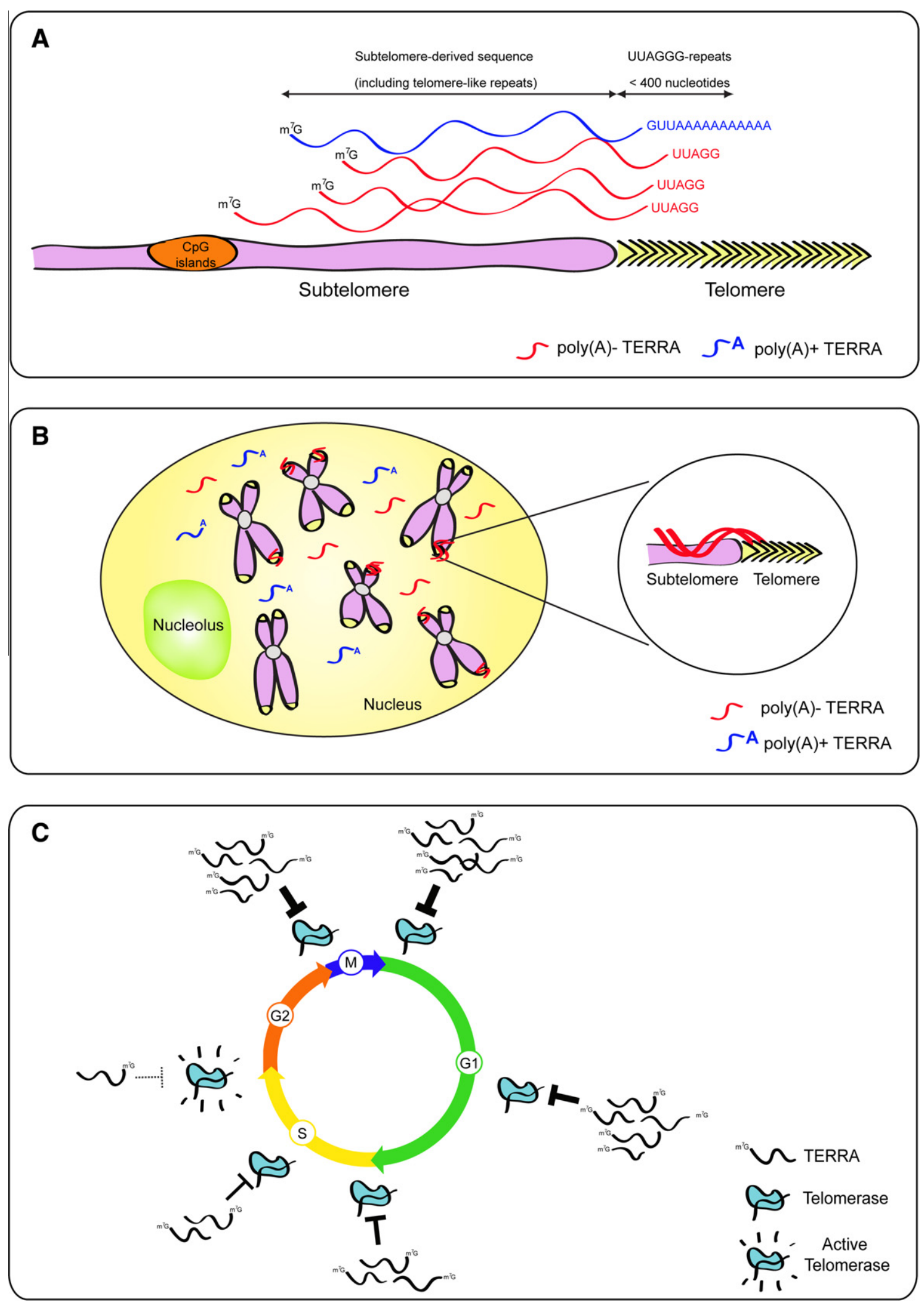

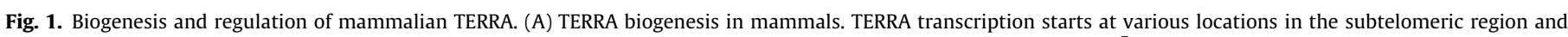

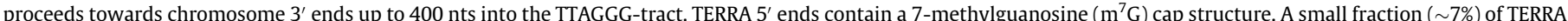

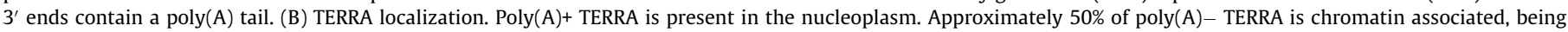

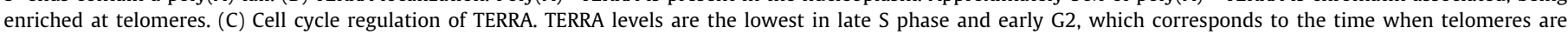
replicated and telomerase is extending chromosome ends. In the model, TERRA represses telomerase outside of $S$ phase.

publication). A minor fraction (7\%) of human TERRA and all yeast TERRA is polyadenylated [9,12].
The higher order structure of TERRA may be determined by TERRA binding proteins and the composition of the elusive TERRA 
ribonucleoprotein particle. Remarkably, however, RNAs with 5'UUAGGG-3' repeats which resemble metazoan TERRA $3^{\prime}$ regions adopt in vitro so-called G-quadruplex structures [13-16]. G-quadruplexes are formed by DNA and RNA molecules that contain repeats of G-rich sequences [17]. In a quadruplex, four guanines are held together in a planar arrangement through eight hydrogen bonds in a Hoogsteen configuration. Several G-quartet planes stack on top of one another forming $\pi-\pi$ interactions, which contribute to G-quadruplex stability. G-quadruplexes are characterized by high thermal stability and they can form under physiological salt and $\mathrm{pH}$ conditions in presence of monovalent cations (preferentially $\mathrm{K}^{+}$). NMR and crystallography experiments with UUAGGG-repeat containing RNA oligonucleotides show formation of Gquadruplex structures in which the four sugar-phosphate strands run $5^{\prime}-3^{\prime}$ in parallel. Notably, CD spectra suggest that TERRA-oligonucleotides can also associate with single stranded DNA TTAGGGrepeat oligonucleotides in trans, forming intermolecular RNA/DNA hybrid G-quadruplex structures [13]. Hybrid TERRA/telomeric DNA structures could potentially form with the telomeric $3^{\prime}$ overhang or at the base of the t-loop in which the G-rich telomeric DNA strand is displaced and therefore is potentially available for TERRA association. Finally, TERRA/telomeric DNA structures may form during lagging strand replication of telomeric DNA and therefore effectively inhibit DNA replication (see below).

\section{TERRA transcription}

TERRA synthesis in mammals and yeast is mediated by RNA polymerase II (pol II) [8,9]. In mammalian cells, however, actinomycin D treatment that inhibits all polymerases causes faster reduction of TERRA levels than specific inhibition of pol II with $\alpha$-amanitin, indicating possible contributions of other RNA polymerases [8]. In S. cerevisiae, the inactivation of pol II by the rpb32 ts allele prevented TERRA accumulation [9]. Pol II is also detected at telomeres in yeast, human and mouse cells $[8,9]$.

Approximately $1 / 4$ of human telomeres contain three specific repetitive elements with $\mathrm{CpG}$-rich sequences in their subtelomeric region [18]. DNA fragments comprising these CpG-islands show promoter activity and may therefore drive TERRA transcription at this subset of telomeres [18]. The cytosine methylation state of these DNA repeats negatively correlates with TERRA abundance [18]. In addition, other telomeric and subtelomeric chromatin hallmarks, such as H3K9 and H4K20 trimethylation, H3K4 di/trimethylation, H3K79 dimethylation, and the overall acetylation state of H3 and H4 tails affect TERRA transcription $[8,12,19,20]$.

TERRA promoter sequences in budding yeast remain to be characterized. However, the major telomere binding protein in budding yeast Rap1 regulates TERRA both at the level of transcription and degradation (Iglesias, N., Redon, S., Dees, M., Lingner, J. and Luke, B., submitted for publication). Budding yeast Rap1 binds directly double-stranded telomeric DNA and it plays a major role in telomere length regulation [21], chromosome end protection [22] and silencing of genes near telomeres, a phenomenon known as telomere position effect (TPE) [23]. The diversity of Rap1 function at telomeres is mediated through the recruitment of Rif (Rif1 and Rif2) and Sir (Sir2, Sir3, and Sir4) complexes via its C-terminal domain [24]. Sir2, recruited to telomeres via Sir4, deacetylates histones and thereby establishes silent chromatin across telomeric loci [24]. Interestingly, in dependency of the presence or absence of $Y^{\prime}$ elements in the subtelomeric region, which are present at about two-thirds of telomeres [25-27], Rap1 uses different protein partners to negatively regulate TERRA. At Y'-containing telomeres, Rap1 regulates TERRA via Rif1 and Rif2 which appear to control the nuclear $5^{\prime}-3^{\prime}$ exonuclease Rat1 and perhaps also transcription (Iglesias, N., Redon, S., Dees, M., Lingner, J. and Luke, B., submitted for publication). In contrast, at telomeres lacking $\mathrm{Y}^{\prime}$ elements, Rap1 promotes TERRA transcrip- tional repression via Sir2/3/4 as well as Rat1-mediated TERRA degradation. Notably, $\mathrm{Y}^{\prime}$ element containing telomeres do not have the classical hallmarks of heterochromatin, characterized by low levels of histone acetylation and high Sir3 occupancy [28,29]. How Rap1 controls TERRA degradation through Rat1 is unclear.

It has to be determined to what extent TERRA regulation by Rap1 is a conserved feature in eukaryotes. In the mouse, Rap1 deletion does not affect TERRA levels [30]. Notably, the sequence conservation of $S$. cerevisiae Rap1 and its orthologs in complex eukaryotes is low [31] and Rap1 interacting partners are different. Complex eukaryotes have no known Sir3, Sir4, or Rif2 homologs, and human Rif1 does not localize to telomeres and is not thought to interact with human Rap1 [32].

Several other chromatin modulators are involved in the regulation of TERRA. Indeed, disturbance of the (sub-)telomeric heterochromatic state in mammalian cells by, e.g. treatment with the histone deacetylase inhibitor trichostatin A or knock-down of histone methyltransferases increases TERRA levels [8,12,33]. Moreover, the histone methyltransferase MLL is involved in TERRA transcription upregulation after telomere uncapping, an effect depending on functional p53 [19]. The yeast ortholog of MLL, known as Set1, plays a role in telomere silencing and its inactivation results in telomere shortening [34]. However, its involvement in TERRA silencing has not been investigated yet. The histone $\mathrm{H} 3$ variant $\mathrm{H} 3.3$ is enriched in the mouse near telomeres and contributes to TERRA repression. Upon deletion of Atrx, a protein required for enrichment of H3.3 at telomeres in embryonic stem cells, TERRA is upregulated 1.7-fold [35].

\section{TERRA $3^{\prime}$ end formation}

The pure telomeric-UUAGGG tract length of TERRA which lacks cytosines was measured by performing TERRA reverse transcription in the absence of dGTP (Porro, A., Feuerhahn, S., Reichenbach, P. and Lingner, J., submitted for publication). This analysis revealed that the $3^{\prime}$ ends of the majority of human TERRA molecules contain $\sim 200$ bases of $5^{\prime}$-UUAGGG-3' repeats with little length heterogeneity. Although post-transcriptional trimming cannot be ruled out, this finding suggests that TERRA transcription does not proceed far into the telomeric tract (Fig. 1A).

Although transcribed by pol II, only $7 \%$ of human TERRA molecules are polyadenylated [12]. The mechanism of TERRA polyadenylation remains to be elucidated as a canonical $5^{\prime}$-AAUAAA- $3^{\prime}$ cleavage and a polyadenylation signal is missing. Canonical polyadenylation increases transcript stability whereas non-canonical poly(A) polymerases are involved in RNA quality control pathways, triggering RNA decay by adding short poly(A) tails to their substrate which then promote exosome-mediated RNA degradation [36,37]. Poly (A) - TERRA has a half life of $\sim 3 \mathrm{~h}$ whereas poly(A)+ TERRA is considerably more stable $\left(t_{1 / 2}>8 \mathrm{~h}\right)$ (Porro, A., Feuerhahn, S., Reichenbach, P. and Lingner, J., submitted for publication). This suggests that the canonical poly(A) polymerase (PAP) is responsible for TERRA polyadenylation in humans. It is notable that poly $(A)+$ and poly $(A)-$ TERRA differ in their termination sites. The $\operatorname{poly}(A)$ tails start with the adenosine within a $5^{\prime}$-UUAGGG-3' repeat whereas poly(A)- TERRA most often terminates in the sequence $5^{\prime}$-UUAGG-3' (73\%) and to a lesser extent (25\%) in the sequence 5'-UUAG-3' (Porro, A., Feuerhahn, S., Reichenbach, P. and Lingner, J., submitted for publication). Altogether, different mechanisms and machineries may be involved in $3^{\prime}$ end formation of poly(A)+ and poly(A) - TERRA populations.

In $S$. cerevisiae, TERRA is polyadenylated by the canonical $\operatorname{poly}(A)$ polymerase Pap1 [9]. In presence of the pap1-1 ts allele, TERRA is quickly disappearing at the restrictive temperature. On the other hand, TRF4 deletion increases TERRA levels indicating that Trf4-dependent polyadenylation does also occur to some ex- 
tent channeling TERRA towards degradation by the nuclear exosome.

\section{Regulation of TERRA: localization and the NMD connection}

Mammalian TERRA is detected exclusively in nuclear fractions, where it partially co-localizes with telomeres not only in interphase cells but also in transcriptionally silent metaphase cells $[7,8]$. Cell extract fractionation experiments revealed that chromatin-associated TERRA is not polyadenylated whereas poly(A)+ TERRA accumulates in the nucleoplasm without binding to telomeric or other chromatin (Porro, A., Feuerhahn, S., Reichenbach, P. and Lingner, J., submitted for publication; Fig. 1B). These data suggest distinct biological functions of different TERRA-pools.

The non-sense-mediated RNA decay (NMD) machinery, which is best known for its role in RNA quality control in the cytoplasm, plays a crucial role in the displacement of TERRA from human telomeres. Depletion of the NMD factors UPF1, SMG1 or EST1A/SMG6 led to increased abundance of TERRA at telomeres [7]. However, neither the overall TERRA degradation rate nor total TERRA expression levels were strongly affected by depletion of these factors. Therefore, UPF1/SMG1/EST1A may trigger specific degradation of the poly(A)- TERRA fraction that is associated with chromatin, rendering the effect undetectable on Northern blots in which total TERRA is analyzed. NMD factors may be directly acting on TERRA at telomeres as they can be detected at telomeres by chromatin immunoprecipitation (ChIP) [7].

In budding yeast, the proteins encoded by the UPF1, UPF2, UPF3, and EBS1 genes promote NMD [38-40]. These proteins localize largely to the cytoplasm. A recent high-throughput analysis using a strain mutated for the NMD component Upf1 reported the accumulation of transcripts from $\mathrm{Y}^{\prime}$-elements and surrounding regions at telomeres [41]. Inactivation of NMD in S. cerevisiae also results in an increase of TERRA transcripts (Iglesias, N. and Lingner, J., unpublished data). However, inactivation of NMD does not decrease the decay rates of TERRA in yeast. This indicates that in budding yeast NMD factors negatively regulate TERRA transcription (Iglesias, N. and Lingner, J., unpublished data). A direct role of yeast NMD factors on TERRA expression would entail that they interact with telomeres as in human cells [42]. In this respect, it is interesting to note that Upf2 has a putative nuclear localization signal (NLS) sequence, which is required for function [43]. Furthermore Upf3, although primarily cytoplasmic, accumulates inside the nucleus when overexpressed [44]. Intriguingly, NMD mutants in budding yeast have short telomeres, which have been attributed to the increased levels of the telomere-capping factors, Stn1 and Ten1 $[45,46]$. It will be interesting to determine whether the shortening of telomeres in NMD mutants is also a consequence of TERRA accumulation and to decipher if NMD factors not only affect TERRA transcription but also impinge on TERRA localization.

\section{A role for TERRA in DNA replication?}

The majority of telomeric DNA is replicated by semiconservative DNA replication and only in telomerase-positive cells, a small portion of the telomere is synthesized de novo at the $3^{\prime}$ end of the chromosome by telomerase. Telomeric DNA is a challenge for the semiconservative DNA replication machinery. In fission yeast, deletion of the double strand DNA binding protein Taz1 leads to stalled replication forks at telomeres and precipitous loss of telomeric DNA that can only be circumvented in the presence of telomerase, which may heal presumed truncated DNA replication intermediates [47]. In mammalian cells, the telomere replication depends on TRF1, which is considered a Taz1-ortholog. Without TRF1, replication forks stall at telomeres and they show a fragile-site phenotype [48].
Replication difficulties at telomeres may be caused by several telomeric features. Since telomeres consist of tandem repeats, slippage events may occur during replication [49]. Furthermore, telomeric repeats can form higher order structures including Gquadruplexes, t-loops and d-loops that may also hinder or prevent replication fork progression [50]. In addition, the heterochromatic and therefore compact nature of telomeres may affect replication progression [51]. Finally, as discussed in the next paragraph TERRA may interfere with replication.

TERRA may hybridize to the C-rich strand of the telomere during replication, particularly at the lagging strand telomere where single stranded C-rich DNA is exposed during lagging strand synthesis. TERRA may also form intermolecular G-quadruplex structures with the G-rich DNA strand of the telomere as discussed above and therefore interfere with replication. This problem may be partially solved through the cell cycle regulation of TERRA. Indeed, qRT-PCR measurements of human TERRA transcripts from four different chromosome ends revealed that TERRA accumulates in early G1 and drops in S phase reaching the lowest expression levels as cells progress from late $S$ to G2 phase (Porro, A., Feuerhahn, S., Reichenbach, P. and Lingner, J., submitted for publication; Fig. 1C). However, a direct interplay between TERRA and DNA replication enzymes and factors at the telomere does exist suggesting additional mechanisms. As discussed above, the $5^{\prime}-3^{\prime}$ DNA and RNA helicase UPF1 as well as other NMD factors regulate telomere abundance of TERRA [7]. Notably, two NMD factors interact with replicative enzymes. UPF1 interacts with DNA polymerase $\delta$, accumulates on chromatin during S-Phase and is essential to complete DNA replication [42,52]. Thus, UPF1 may ensure proper telomere replication by displacing or locally degrading TERRA from telomeres for progression of the replication fork, thereby avoiding unwanted annealing of TERRA to single-stranded telomeric DNA. The NMD factor EST1A/SMG6 physically interacts with telomerase and it might play a role in liberating telomerase from TERRA-mediated inhibition [53-55]. Interestingly, the cells from patients suffering from the Immunodeficiency Centromeric Instability and Facial abnormalities type I syndrome (ICF) in which one DNMT3b allele is mutated [33] have increased TERRA levels. Similar to cells in which NMD function is impaired, telomeres are lost at a high rate in ICF cells. In addition, increased TERRA levels correlate in ICF cells with decreased subtelomeric heterochromatin formation as well as untimed replication onset.

TERRA was reported to physically interact with TRF1 and TRF2 in order to recruit the ORC2 protein to telomeres [56]. Although origin firing within telomeric repeats is a rare event [48], it seems conceivable that ORC proteins at telomeres assist telomere replication. Transfection of siRNAs that were designed to target TERRA decreased telomere-association of ORC2 [56]. However, in the Deng et al. paper, the TERRA signal on the Northern blot was smaller than $1 \mathrm{~kb}$ which is considerably smaller than what has been reported for TERRA in studies from several other laboratories $[7,8,30,35,57]$. Thus, it seems that a different RNA population may have been assessed by Deng et al. It is also interesting to note that in previous studies the transfection of short telomeric DNA oligonucleotides led to severe perturbation of telomere function [58]. Overall, transfection of oligonucleotides with telomeric sequence may have multiple direct and indirect effects on telomere structure and function. It remains an important challenge for the future to unequivocally identify TERRA functions.

\section{TERRA and heterochromatin formation}

Telomeres as well as subtelomeres are enriched for several heterochromatic marks including H3K9 and H4K20 trimethylation imposed by histone methyl transferases (HMTs), heterochromatin Protein 1 (HP1) as well as hypoacetylated histones [59,60]. More- 
over, subtelomeric DNA is heavily methylated by DNA methyltransferases (DNMTs) in human cells [33]. Other non-coding RNAs have been functionally linked to heterochromatin formation including Xist and Piwi [61-63] and it is tempting to speculate that TERRA is involved in telomeric heterochromatin formation. A regulatory loop connecting telomere length, TERRA abundance as well as telomeric heterochromatinization has been suggested because of the inverse correlation between telomere length and density of heterochromatin marks and the correlation between TERRA levels and telomere length [8].

Diminished H3K9 methylation at telomeres was reported upon transfection of siRNAs against TERRA [56]. However, as discussed above the interpretation of these experiments may not be straightforward. Nevertheless, in the same study, it was shown by RNA ChIP that TERRA associates with HP1alpha and H3K9me3, reinforcing the idea of a link between TERRA and heterochromatin. It remains still to be established if TERRA level changes are cause or consequence for heterochromatic effects.

TERRA is also detected in Arabidopsis thaliana [10]. Moreover, in this species, as well as in S. cerevisiae, TERRA antisense transcripts, termed ARRET, have recently been discovered [9,10]. Arabidopsis TERRA and ARRET are not only derived from telomeres but are mostly derived from centromeric regions, which contain remnants of telomeric DNA. siRNAs derived from plant TERRA and ARRET transcripts were detected and they are generated in dependency of the RNA-dependent DNA methylation (RdDM) pathway, which is a plant-specific mechanism that utilizes siRNAs to guide DNA methyltransferases to asymmetric cytosines (CNN) [10]. TERRAderived siRNA is associated with AGO4, which is part of the effector complex that mediated CNN methylation of telomeric DNA. In summary, a causal role of TERRA for heterochromatin formation is most clearly emerging in plants [10]. Small RNAs containing telomeric sequences have also been reported for mouse embryonic stem cells but their function remains unknown [64].

\section{Regulation of telomerase}

Telomerase is thought to act after conservative DNA replication [65] extending the $3^{\prime}$ end of chromosomes in order to counteract telomere shortening that occurs due to the end replication problem and nucleolytic processing of chromosome ends. Several lines of evidence suggest that TERRA may act as a direct regulator of telomerase $[8,66]$. TERRA molecules resemble in sequence the telomeric DNA substrate. Thus they contain telomeric repeats (e.g. 5'UUAGGG-3' repeats in mammals) near their $3^{\prime}$ ends, which are complementary to the template sequence of telomerase RNA. A fraction of endogenous TERRA is bound to human telomerase in cell extracts [66]. Furthermore, using in vitro reconstituted telomerase and synthetic TERRA molecules it was demonstrated that the 5'-UUAGGG-3' repeats of TERRA base-pair with the RNA template of the telomerase RNA moiety [66]. In addition TERRA contacts the telomerase reverse transcriptase (TERT) protein subunit independently of hTR. The TERRA-hTERT interaction site remains to be characterized. Overall, the affinity of telomerase for TERRA is remarkable as it exceeds the affinity for telomeric DNA. In vitro studies further demonstrate that TERRA is not used as a telomerase substrate. Instead, TERRA acts as a potent competitive inhibitor for telomeric DNA in addition to exerting an uncompetitive mode of inhibition. The observed mixed inhibition by TERRA indicates that TERRA can bind to telomerase even while bound to the telomere substrate [66]. This may be explainable through a direct hTERT-TERRA interaction, which may be distinct from the previously reported direct TERT-DNA contacts that are elicited through the so-called anchor site [67].

As already discussed, TERRA is displaced and/or degraded at telomeres by NMD factors which physically interact with the telomeric chromatin [7]. Among these factors, EST1A/SMG6 was also identified through its sequence similarity with the $S$. cerevisiae telomerase associated protein Est1 and human EST1A/SMG6, as yeast Est1 physically interacts with telomerase [53-55].

Genetic experiments in $S$. cerevisiae provide evidence that TERRA regulates telomerase in vivo. In the rat1-1 mutant background in which the function of the $5^{\prime}-3^{\prime}$ exonuclease Rat 1 is reduced, TERRA is upregulated and telomeres are shorter than in wild type cells due to impairment of telomerase-mediated telomere elongation [9]. Further support for the role of TERRA in inhibiting telomerase in vivo stems from an observation that forced telomere transcription (through the use of the strong Gal-promoter) leads to telomere shortening of the transcribed telomere in cis [68].

\section{Perspective}

Feedback loops may regulate telomere transcription, chromatin structure and telomere length. Telomere transcription and TERRA may promote at the same time heterochromatization and prevent telomerase-mediated telomere elongation. Reinforcement of telomeric heterochromatin should in turn reduce telomere transcription, which again may weaken the heterochromatic state. Similarly, it is possible that upon telomerase-mediated telomere elongation telomere transcription is reinforced to stop further elongation. In addition, telomere transcription may respond to other local cues at the telomere or more global stress situations in order to modulate chromosome end structure and repress telomerase. At this stage it remains rather unclear under which physiological circumstances TERRA is induced and if the purpose is indeed telomerase repression or the modulation of chromatin structure. The cell cycle regulation of TERRA and the timing of telomere elongation would be consistent with a role in repressing telomerase outside of S-phase in a cell cycle dependent manner (Fig. 1C). Interestingly, distinct TERRA populations can be distinguished through the nature of their $3^{\prime}$ ends and their association with chromatin. Distinct TERRA ribonucleoprotein particles might exert different functions. If non-chromatin-associated TERRA was associating with telomerase it would elicit an overall inhibition of the enzyme whereas telomere-associated TERRA should act specifically in cis.

It will be important to develop systems that allow genetic manipulation of TERRA transcription, localization and assembly into the elusive RNP particles in order to learn about its functions. Elucidation of TERRA biogenesis may provide the necessary tools that allow perturbation of TERRA. Identification of TERRA binding proteins may give hints on which cellular activities may be recruited or locally sequestered by this RNA. In addition, it may allow identification of protein factors that are important for subnuclear localization or turnover. An additional challenge will be to understand possible interactions of TERRA with telomeric DNA. In the rat1-1 yeast mutants, TERRA-DNA hybrids seem to form, which can be overcome through overexpression of RNase $\mathrm{H}$, which degrades RNA in DNA/RNA hybrids [9]. However, it is unclear if TERRA/DNA hybrids also occur in wild type cells. It will also be a challenge to assess possible G-quadruplex interactions between TERRA and telomeric DNA in trans. Notably, TERRA is only one of several recent examples that testify the amazing structural and functional complexity that is unfolding at telomeres. The closer we get the more can be seen. This is not the end. It is not even the beginning of the end. But it is, perhaps, the end of the beginning.

\section{Acknowledgments}

Supported by an EMBO Long-Term Fellowship to A. Porro and grants to J.L. from the Swiss National Science Foundation, the European Community's Seventh Framework Programme FP7/20072011 (grant agreement number 200950) and a European Research 
Council advanced investigator grant (grant agreement number 232812).

\section{References}

[1] de Lange, T. (2009) How telomeres solve the end-protection problem. Science $326,948-952$

[2] Greider, C.W. and Blackburn, E.H. (1985) Identification of a specific telomere terminal transferase activity in Tetrahymena extracts. Cell 43, 405-413.

[3] Greider, C.W. and Blackburn, E.H. (1989) A telomeric sequence in the RNA of Tetrahymena telomerase required for telomere repeat synthesis. Nature 337, 331-337.

[4] Lingner, J., Hughes, T.R., Shevchenko, A., Mann, M., Lundblad, V. and Cech, T.R. (1997) Reverse transcriptase motifs in the catalytic subunit of telomerase. Science 276, 561-567.

[5] Bodnar, A.G. et al. (1998) Extension of life-span by introduction of telomerase into normal human cells. Science 279, 349-352.

[6] Kim, N.W. et al. (1994) Specific association of human telomerase activity with immortal cells and cancer. Science 266, 2011-2015.

[7] Azzalin, C.M., Reichenbach, P., Khoriauli, L., Giulotto, E. and Lingner, J. (2007) Telomeric repeat containing RNA and RNA surveillance factors at mammalian chromosome ends. Science 318, 798-801.

[8] Schoeftner, S. and Blasco, M.A. (2008) Developmentally regulated transcription of mammalian telomeres by DNA-dependent RNA polymerase II. Nat. Cell Biol. 10, 228-236.

[9] Luke, B., Panza, A., Redon, S., Iglesias, N., Li, Z. and Lingner, J. (2008) The Rat1p $5^{\prime}$ to $3^{\prime}$ exonuclease degrades telomeric repeat-containing RNA and promotes telomere elongation in Saccharomyces cerevisiae. Mol. Cell 32, 465-477.

[10] Vrbsky, J., Akimcheva, S., Watson, J.M., Turner, T.L., Daxinger, L., Vyskot, B., Aufsatz, W. and Riha, K. (2010) SiRNA-mediated methylation of Arabidopsis telomeres. PLoS Genet. 6, e1000986.

[11] Luke, B. and Lingner, J. (2009) TERRA: telomeric repeat-containing RNA. EMBO J. 28, 2503-2510.

[12] Azzalin, C.M. and Lingner, J. (2008) Telomeres: the silence is broken. Cell Cycle 7, 1161-1165.

[13] Xu, Y., Kimura, T. and Komiyama, M. (2008) Human telomere RNA and DNA form an intermolecular G-quadruplex. Nucleic Acids Symp. Ser. (Oxf) 169, 70

[14] Martadinata, H. and Phan, A.T. (2009) Structure of propeller-type parallelstranded RNA G-quadruplexes, formed by human telomeric RNA sequences in $\mathrm{K}+$ solution. J. Am. Chem. Soc. 131, 2570-2578.

[15] Randall, A. and Griffith, J.D. (2009) Structure of long telomeric RNA transcripts: the G-rich RNA forms a compact repeating structure containing G-quartets. J. Biol. Chem. 284, 13980-13986.

[16] Collie, G.W., Haider, S.M., Neidle, S. and Parkinson, G.N. (2010) A crystallographic and modelling study of a human telomeric RNA (TERRA) quadruplex. Nucleic Acids Res., Epub ahead of print.

[17] Lipps, H.J. and Rhodes, D. (2009) G-quadruplex structures: in vivo evidence and function. Trends Cell Biol. 19, 414-422.

[18] Nergadze, S.G., Farnung, B.O., Wischnewski, H., Khoriauli, L., Vitelli, V., Chawla R., Giulotto, E. and Azzalin, C.M. (2009) CpG-island promoters drive transcription of human telomeres. RNA 15, 2186-2194.

[19] Caslini, C., Connelly, J.A., Serna, A., Broccoli, D. and Hess, J.L. (2009) MLL associates with telomeres and regulates telomeric repeat-containing RNA transcription. Mol. Cell. Biol. 29, 4519-4526.

[20] Jones, B. et al. (2008) The histone H3K79 methyltransferase Dot1L is essential for mammalian development and heterochromatin structure. PLoS Genet. 4 e1000190.

[21] Marcand, S., Gilson, E. and Shore, D. (1997) A protein-counting mechanism for telomere length regulation in yeast. Science 275, 986-990.

[22] Marcand, S., Pardo, B., Gratias, A., Cahun, S. and Callebaut, I. (2008) Multiple pathways inhibit NHEJ at telomeres. Genes Dev. 22, 1153-1158.

[23] Gottschling, D.E., Aparicio, O.M., Billington, B.L. and Zakian, V.A. (1990) Position effect at $S$. cerevisiae telomeres: reversible repression of Pol Il transcription. Cell 63, 751-762.

[24] Shore, D. (2001) Telomeric chromatin: replicating and wrapping up chromosome ends. Curr. Opin. Genet. Dev. 11, 189-198.

[25] Chan, C.S. and Tye, B.K. (1983) Organization of DNA sequences and replication origins at yeast telomeres. Cell 33, 563-573.

[26] Walmsley, R.W., Chan, C.S., Tye, B.K. and Petes, T.D. (1984) Unusual DNA sequences associated with the ends of yeast chromosomes. Nature 310,157 160.

[27] Louis, E.J. (1995) The chromosome ends of Saccharomyces cerevisiae. Yeast 11, 1553-1573.

[28] Pryde, F.E. and Louis, E.J. (1999) Limitations of silencing at native yeast telomeres. EMBO J. 18, 2538-2550.

[29] Zhu, X. and Gustafsson, C.M. (2009) Distinct differences in chromatin structure at subtelomeric $X$ and $\mathrm{Y}^{\prime}$ elements in budding yeast. PLoS One 4, e6363.

[30] Sfeir, A., Kabir, S., van Overbeek, M., Celli, G.B. and de Lange, T. (2010) Loss of Rap1 induces telomere recombination in the absence of NHEJ or a DNA damage signal. Science 327, 1657-1661.

[31] Li, B., Oestreich, S. and de Lange, T. (2000) Identification of human Rap1: implications for telomere evolution. Cell 101, 471-483.

[32] Silverman, J., Takai, H., Buonomo, S.B., Eisenhaber, F. and de Lange, T. (2004) Human Rif1, ortholog of a yeast telomeric protein, is regulated by ATM and 53BP1 and functions in the S-phase checkpoint. Genes Dev. 18, 2108-2119.
[33] Yehezkel, S., Segev, Y., Viegas-Pequignot, E., Skorecki, K. and Selig, S. (2008) Hypomethylation of subtelomeric regions in ICF syndrome is associated with abnormally short telomeres and enhanced transcription from telomeric regions. Hum. Mol. Genet. 17, 2776-2789.

[34] Dehe, P.M. and Geli, V. (2006) The multiple faces of Set1. Biochem. Cell Biol. 84, 536-548.

[35] Goldberg, A.D. et al. (2010) Distinct factors control histone variant H3.3 localization at specific genomic regions. Cell 140, 678-691.

[36] LaCava, J., Houseley, J., Saveanu, C., Petfalski, E., Thompson, E., Jacquier, A. and Tollervey, D. (2005) RNA degradation by the exosome is promoted by a nuclear polyadenylation complex. Cell 121, 713-724.

[37] Vanacova, S. et al. (2005) A new yeast poly(A) polymerase complex involved in RNA quality control. PLoS Biol. 3, e189.

[38] Isken, O. and Maquat, L.E. (2007) Quality control of eukaryotic mRNA: safeguarding cells from abnormal mRNA function. Genes Dev. 21, 1833-1856.

[39] Baker, K.E. and Parker, R. (2004) Nonsense-mediated mRNA decay: terminating erroneous gene expression. Curr. Opin. Cell Biol. 16, 293-299.

[40] Luke, B., Azzalin, C.M., Hug, N., Deplazes, A., Peter, M. and Lingner, J. (2007) Saccharomyces cerevisiae Ebs1p is a putative ortholog of human Smg7 and promotes nonsense-mediated mRNA decay. Nucleic Acids Res. 35, 76887697.

[41] Sayani, S., Janis, M., Lee, C.Y., Toesca, I. and Chanfreau, G.F. (2008) Widespread impact of nonsense-mediated mRNA decay on the yeast intronome. Mol. Cell $31,360-370$

[42] Azzalin, C.M. and Lingner, J. (2006) The human RNA surveillance factor UPF1 is required for $S$ phase progression and genome stability. Curr. Biol. 16, 433-439.

[43] He, F., Brown, A.H. and Jacobson, A. (1996) Interaction between Nmd2p and Upf1p is required for activity but not for dominant-negative inhibition of the nonsense-mediated mRNA decay pathway in yeast. RNA 2, 153-170.

[44] Shirley, R.L., Lelivelt, M.J., Schenkman, L.R., Dahlseid, J.N. and Culbertson, M.R. (1998) A factor required for nonsense-mediated mRNA decay in yeast is exported from the nucleus to the cytoplasm by a nuclear export signal sequence. J. Cell Sci. 111 (Pt 21), 3129-3143.

[45] Dahlseid, J.N. et al. (2003) MRNAs encoding telomerase components and regulators are controlled by UPF genes in Saccharomyces cerevisiae. Eukaryot. Cell 2, 134-142.

[46] Enomoto, S., Glowczewski, L., Lew-Smith, J. and Berman, J.G. (2004) Telomere cap components influence the rate of senescence in telomerase-deficient yeast cells. Mol. Cell. Biol. 24, 837-845.

[47] Miller, K.M., Rog, O. and Cooper, J.P. (2006) Semi-conservative DNA replication through telomeres requires Taz1. Nature 440, 824-828.

[48] Sfeir, A., Kosiyatrakul, S.T., Hockemeyer, D., MacRae, S.L., Karlseder, J., Schildkraut, C.L. and de Lange, T. (2009) Mammalian telomeres resemble fragile sites and require TRF1 for efficient replication. Cell 138, 90-103.

[49] Mirkin, S.M. (2006) DNA structures, repeat expansions and human hereditary disorders. Curr. Opin. Struct. Biol. 16, 351-358.

[50] Gilson, E. and Geli, V. (2007) How telomeres are replicated. Nat. Rev. Mol. Cell Biol. 8, 825-838.

[51] McCord, R.A. and Broccoli, D. (2008) Telomeric chromatin: roles in aging, cancer and hereditary disease. Mutat. Res. 647, 86-93.

[52] Carastro, L.M., Tan, C.K., Selg, M., Jack, H.M., So, A.G. and Downey, K.M. (2002) Identification of delta helicase as the bovine homolog of HUPF1: demonstration of an interaction with the third subunit of DNA polymerase delta. Nucleic Acids Res. 30, 2232-2243.

[53] Snow, B.E., Erdmann, N., Cruickshank, J., Goldman, H., Gill, R.M., Robinson, M.O. and Harrington, L. (2003) Functional conservation of the telomerase protein Est1p in humans. Curr. Biol. 13, 698-704.

[54] Redon, S., Reichenbach, P. and Lingner, J. (2007) Protein RNA and proteinprotein interactions mediate association of human EST1A/SMG6 with telomerase. Nucleic Acids Res. 35, 7011-7022.

[55] Reichenbach, P., Hoss, M., Azzalin, C.M., Nabholz, M., Bucher, P. and Lingner, J. (2003) A human homolog of yeast Est1 associates with telomerase and uncaps chromosome ends when overexpressed. Curr. Biol. 13, 568-574.

[56] Deng, Z., Norseen, J., Wiedmer, A., Riethman, H. and Lieberman, P.M. (2009) TERRA RNA binding to TRF2 facilitates heterochromatin formation and ORC recruitment at telomeres. Mol. Cell 35, 403-413.

[57] Ho, C.Y., Murnane, J.P., Yeung, A.K., Ng, H.K. and Lo, A.W. (2008) Telomeres acquire distinct heterochromatin characteristics during siRNA-induced RNA interference in mouse cells. Curr. Biol. 18, 183-187.

[58] Li, G.Z., Eller, M.S., Firoozabadi, R. and Gilchrest, B.A. (2003) Evidence that exposure of the telomere $3^{\prime}$ overhang sequence induces senescence. Proc. Natl. Acad. Sci. USA 100, 527-531.

[59] Benetti, R., Gonzalo, S., Jaco, I., Schotta, G., Klatt, P., Jenuwein, T. and Blasco, M.A. (2007) Suv4-20h deficiency results in telomere elongation and derepression of telomere recombination. J. Cell Biol. 178, 925-936.

[60] Blasco, M.A. (2007) The epigenetic regulation of mammalian telomeres. Nat. Rev. Genet. 8, 299-309.

[61] Zhao, J., Sun, B.K., Erwin, J.A., Song, J.J. and Lee, J.T. (2008) Polycomb proteins targeted by a short repeat RNA to the mouse X chromosome. Science 322, 750-756.

[62] Kuramochi-Miyagawa, S. et al. (2008) DNA methylation of retrotransposon genes is regulated by Piwi family members MILI and MIWI2 in murine fetal testes. Genes Dev. 22, 908-917.

[63] Aravin, A.A., Sachidanandam, R., Bourc'his, D., Schaefer, C., Pezic, D., Toth, K.F. Bestor, T. and Hannon, G.J. (2008) A piRNA pathway primed by individual 
transposons is linked to de novo DNA methylation in mice. Mol. Cell 31, 785799.

[64] Cao, F., Li, X., Hiew, S., Brady, H., Liu, Y. and Dou, Y. (2009) Dicer independent small RNAs associate with telomeric heterochromatin. RNA 15, 1274-1281.

[65] Zhao, Y., Sfeir, A.J., Zou, Y., Buseman, C.M., Chow, T.T., Shay, J.W. and Wright, W.E. (2009) Telomere extension occurs at most chromosome ends and is uncoupled from fill-in in human cancer cells. Cell 138, 463-475.
66] Redon, S., Reichenbach, P. and Lingner, J. (2010) The non-coding RNA TERRA is a natural ligand and direct inhibitor of human telomerase. Nucleic Acids Res. Epub ahead of print.

[67] Zaug, A.J., Podell, E.R. and Cech, T.R. (2008) Mutation in TERT separate processivity from anchor-site function. Nat. Struct. Mol. Biol. 15, 870-872.

[68] Sandell, L.L., Gottschling, D.E. and Zakian, V.A. (1994) Transcription of a yeast telomere alleviates telomere position effect without affecting chromosome stability. Proc. Natl. Acad. Sci. USA 91, 12061-12065. 